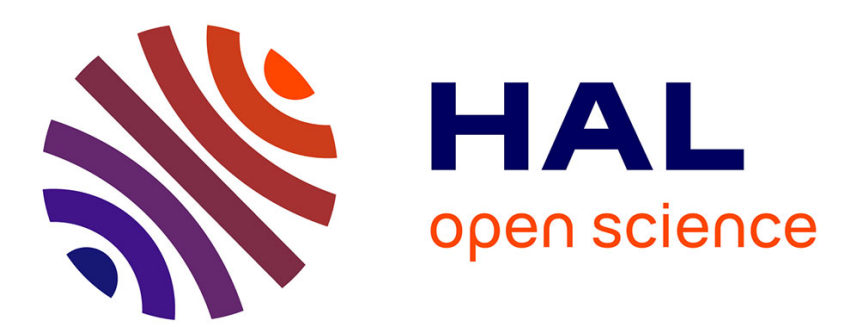

\title{
Décomposition isotherme et anisotherme de l'austenite en ferrite dans des aciers de base 9Cr1Mo: aspects cinétiques et microstructuraux
}

\author{
J.-C. Brachet
}

\section{- To cite this version:}

J.-C. Brachet. Décomposition isotherme et anisotherme de l'austenite en ferrite dans des aciers de base 9Cr1Mo: aspects cinétiques et microstructuraux. Journal de Physique IV Proceedings, 1994, 04 (C3), pp.C3-83-C3-91. 10.1051/jp4:1994311 . jpa-00252508

HAL Id: jpa-00252508 https://hal.science/jpa-00252508

Submitted on 1 Jan 1994

HAL is a multi-disciplinary open access archive for the deposit and dissemination of scientific research documents, whether they are published or not. The documents may come from teaching and research institutions in France or abroad, or from public or private research centers.
L'archive ouverte pluridisciplinaire HAL, est destinée au dépôt et à la diffusion de documents scientifiques de niveau recherche, publiés ou non, émanant des établissements d'enseignement et de recherche français ou étrangers, des laboratoires publics ou privés. 


\title{
Décomposition isotherme et anisotherme de l'austenite en ferrite dans des aciers de base 9 Cr1Mo : aspects cinétiques et microstructuraux
}

\author{
J.-C. BRACHET
}

CEA/CEREM/DTM/SRMA, CE Saclay, 91191 Gif-sur-Yvette cedex, France

\begin{abstract}
:
Isothermal and anisothermal (under-cooling) phase transformations from austenite have been studied in a $9 \mathrm{Cr} 1 \mathrm{Mo}$ alloy (EM10) martensitic steel. In isothermal conditions, this steel displays typical C-curve TTT diagram for temperatures ranging from 600 to $800^{\circ} \mathrm{C}$. For lower temperatures, no bainitic reaction seems to occur for isothermal anneal times up to $500 \mathrm{~h}$. The isothermal austenite to ferrite reaction has been described by the JOHNSON-MEHL-AVRAMI relation with the introduction of a "nucleation time" $\left(t_{0}\right): Y(\alpha)=1-\exp \left\{-\left[K\left(t-t_{0}\right)\right]^{N}\right\}$ with $K=1 /\left(t_{63.2 \%}-t_{0}\right)$. The half-reaction time seems to depend on the mean austenite grain size as predicted by the CAHN relation $: t_{1 / 2}=K d^{m}$, where the experimental $m$ values can be related to the ferrite nucleation conditions. From microstructure and kinetic considerations, we can distinguish two domains of the isothermal transformation temperature $\left(\theta_{\mathrm{i} . t}\right)$ where the austenite to ferrite transformation is quite different :

* For $\theta_{i . t .}>700^{\circ} \mathrm{C}:$ Microstructure is characterized by equiaxed grains of ferrite showing coarse "pearlitic" $\mathrm{M}_{23} \mathrm{C}_{6}$ carbide morphology. Ferrite nucleation seems to occur preferentially on austenitic corner boundaries, without early saturation of the potential nucleation sites during the isothermal ferrite growth.

* For $\theta_{i . t .}<700^{\circ} \mathrm{C}$ : The austenite to ferrite transformation is initiated on all the austenitic grain boundaries. Nucleation occurs with "interphase" precipitation of $\mathrm{M}_{23} \mathrm{C}_{6}$ carbides. After about $20 \%$ of transformed austenite, $\mathrm{M}_{2} \mathrm{X}$ carbonitride precipitation occurs. Finally, at the end of the reaction, there is no more precipitation. Complementary experiments using Martensite start temperature, micro-hardness of residual martensite and Thermo-electric Power measurements for partial transformation of austenite to ferrite at 650,725 and $785^{\circ} \mathrm{C}$, show that there is a quite fast carbon depletion of austenite during the early stages of the isothermal reaction at low temperatures $\left(\approx 650^{\circ} \mathrm{C}\right)$.

Finally, the results obtained under isothermal conditions are used and to discuss the kinetics and microstructure evolutions during anisothermal under-cooling phase transformation from austenite.
\end{abstract}




\section{INTRODUCTION:}

Les aciers martensitiques $9 \mathrm{Cr} 1 \mathrm{Mo}$ sont étudiés depuis de nombreuses années afin d'être utilisés comme matériau de structure des réacteurs nucléaires ([1] à [4]), en particulier pour la fabrication des tubes hexagonaux (TH) de l'assemblage combustible de la filière à neutrons rapides ([5] à [8]). En outre, ils sont envisagés pour la réalisation de la première paroi des futurs réacteurs a fusion contrôlée ([9] à [11]). Ces aciers allient une résistance mécanique élevée et une ductilité satisfaisante aux températures de service $\left(400^{\circ} \mathrm{C}\right.$ $600^{\circ} \mathrm{C}$ ). Ils présentent une très bonne stabilité dimensionelle et des propriétés mécaniques usuelles (traction, résilience) sous flux neutronique, ainsi qu'une bonne compatibilité avec le Sodium liquide. Toutefois, l'élaboration, la transformation et la soudabilité de ces matériaux sont des paramètres difficiles à maîtriser. Il est donc important de mieux cerner la métallurgie physique de ces matériaux, en particulier d'appréhender les transformations de phases et les évolutions microstructurales associées qui se développent lors de différents cycles thermiques.

Dans cet esprit, cette étude s'intéresse aux réactions de décomposition isotherme et anisotherme de la phase de haute température austénitique. L'alliage étudié est un acier à $9 \% \mathrm{Cr}-1 \% \mathrm{Mo}-0.1 \% \mathrm{C}-0.025 \% \mathrm{~N}$ (\% en poids) de dénomination commerciale EM10. Cette nuance constitue le matériau de référence pour la fabrication des TH des Réacteurs à Neutrons Rapides Français.

\section{DECOMPOSITION ISOTHERME DE L:AUSTENITE:}

\subsection{Aspects cinétiques :}

Après austénitisation d'une heure à $1030^{\circ} \mathrm{C}$, l'acier étudié présente un diagramme TTT avec une allure typique en " $\mathrm{C}$ " comme il est présenté sur la figure 1. Ce diagramme est caractéristique de la transformation isotherme \{austénite $=>$ \{ferrite\}. Aucune transformation de type bainitique n'a pu être observée jusqu'à des temps de maintien isotherme de $500 \mathrm{~h}$, dans le domaine de temperature intermédiaire entre le domaine ferritique observe $\left(\theta=600-800^{\circ} \mathrm{C}\right)$ et le domaine martensitique $\left(\mathrm{Ms} \approx 400^{\circ} \mathrm{C}\right.$ et $\left.\mathrm{Mf} \approx 230^{\circ} \mathrm{C}\right)$. Ce phénomène de retard a la transformation bainitique a déjà été observé dans les aciers fortement alliés au Chrome ou au Molybdène; l'un des mécanismes proposés pour expliquer ce phénomène est un drainage des atomes substitutionnels (Cr, Mo) "solute drag-like effect" par l'interface \{ferrite\}-\{austénite\} en croissance, d'ou un fort ralentissement de l'avancée de la réaction isotherme ([12] à [15]).

La décomposition isotherme de l'austénite en ferrite, mesurée entre $625^{\circ} \mathrm{C}$ et $775^{\circ} \mathrm{C}$, est correctement décrite par l'équation intégrée de Jonhson-Mehl-Avrami (JMA) [16], auquelle a été ajoutée un paramètre ajustable supplémentaire (en plus de l'exposant du temps) que l'on peut définir comme un temps d'incubation tinc.

$$
\mathrm{Y}(\alpha)=1-\exp \left(\left[-\mathrm{K}\left(\mathrm{t}-\mathrm{t}_{\text {inc }}\right)\right]^{\mathrm{N}}\right) \text { avec } \mathrm{K}=1 /\left(\mathrm{t}_{63}, 28-\mathrm{t}_{\text {inc }}\right)
$$

Les valeurs expérimentales de l'exposant du temps $(\mathrm{N})$ diminuent de 4 à $2.5 \pm 0.3$, lorsque la température de transformation isotherme $\left(\theta_{\mathfrak{i}}\right)$ diminue de $750^{\circ} \mathrm{C}$ ("nez" du diagramme TTT) à $625^{\circ} \mathrm{C}$. D'autre part, nous avons utilisé deux méthodes explicitées en détail par J. BURKE [17], dites du "temps de réaction fractionnaire" et de la "variation de vitesse", pour déterminer des énergies d'activation apparentes ( $E_{\text {app. }}$ ) caractéristiques des cinétiques isothermes expérimentales. Les calculs conduisent à des valeurs comprises entre 160 et $335 \mathrm{~kJ} / \mathrm{mol}$. Ces valeurs seraient plus proches de celles relatives a la diffusion des atomes substitutionnels $(\mathrm{Cr}, \mathrm{Mo})$ qu'à celles relatives à la diffusion des interstitiels ( $\mathrm{C}$ et $\mathrm{N}$ ). Elles dépendent du degré d'avancement de la réaction isotherme. Ceci signifie que les courbes ne sont pas "isocinétiques" [17] dans le domaine de températures considéré. Un développement et une discussion plus détaillée de ce problème particulier figurent dans [7]. 


\subsection{Aspects microstructuraux :}

Il existe deux domaines de températures pour lesquels les réactions de précipitation qui accompagnent la transformation isotherme \{austénite $=>$ fferrite\} sont différentes. Pour les températures $\left(\theta_{i}\right)$ correspondant au "nez" de la courbe TTT $\left(725\right.$ a $\left.775^{\circ} \mathrm{C}\right)$, la précipitation adopte une morphologie de type "perlitique" (fig. 1-bis). Celle-ci est essentiellement constituée de carbures de Chrome du type $\mathrm{M}_{23} \mathrm{C}_{6}$. Pour les plus basses temperatures $\left(625-700^{\circ} \mathrm{C}\right)$, la précipitation est localisée au voisinage de l'ancien joint de grain austénitique (fig. 1-bis). Elle se présente sous la forme de fins carbures $\mathrm{M}_{23} \mathrm{C}_{6}$ plus ou moins alignés, typique d'une précipitation de type "interphase" ([18] à [21]). On peut noter que ces précipités présentent des relations d'orientation cristallographique avec la matrice [7]. D'autre part, pour des nuances d'acier $9 \mathrm{Cr} 1$ Mo contenant du Vanadium (0.2\%), ils durcissent sensiblement la matrice ferritique [8].

La figure 2 présente deux micrographies optiques caractéristiques de la structure ferritomartensitique obtenue après décomposition isotherme partielle de l'austénite à $725^{\circ} \mathrm{C}$ et $650^{\circ} \mathrm{C}$. On constate qu'à $725^{\circ} \mathrm{C}$, la ferrite germe préférentiellement sur les joints triples austénitiques, alors qu'à $650^{\circ} \mathrm{C}$, l'ensemble des joints austénitiques sont saturés par les germes de ferrite très tôt au cours de la réaction isotherme. Cette tendance est confirmée par une estimation à l'aide de l'analyse d'images du nombre de grains $\alpha$ apparus pour differents taux de transformation a $725^{\circ} \mathrm{C}$, comme le montre la figure 3 . Cette figure souligne que la vitesse de germination de la ferrite tout au long de la réaction isotherme semble relativement constante à cette température; ceci serait d'ailleurs tout à fait coherent vis à vis de la valeur expérimentale de l'exposant du temps de l'équation de JMA : N=4, trouvée à cette température [22]. Enfin, la variation du temps de demi-réaction isotherme $\left(t_{1 / 2}\right)$ a été déterminée en fonction de la taille moyenne du grain de la phase "mère" austénitique, pour différentes conditions d'austénitisation. Les résultats expérimentaux montrent que, conformément au modele proposé par J. W. CAHN [23], $\mathrm{t}_{1 / 2}$ varie selon une loi puissance en fonction du diametre du grain austénitique (d) : $t_{1 / 2}=K d^{M}$ (fig. 4).

La figure 5 décrit schématiquement, et à l'aide de micrographies électroniques effectuées sur répliques extractives au carbone, la séquence de précipitation qui accompagne la décomposition isotherme de l'austénite à $650^{\circ} \mathrm{C}$. L'évolution observée est la suivante:

(1) germination et début de la croissance de la ferrite avec précipitation dense de carbures interphases $\mathrm{M}_{23} \mathrm{C}_{6}$;

(2) après environ 10 à $20 \%$ d'austénite transformée, la précipitation de $\mathrm{M}_{23} \mathrm{C}_{6}$ fait place à un debut de précipitation de carbonitrures de Chrome du type $\mathrm{M}_{2} \mathrm{X}$;

(3) la fin de la réaction a lieu avec très peu de précipitation associée.

Un mécanisme schématique est proposé sur la figure 5 pour décrire l'évolution du profil de concentration en solution solide du carbone $(C)$ et de l'azote $(N)$, en amont de l'interface \{ferrite\}-\{austénite\} en croissance. Celui-ci suppose que l'austénite non transformée s'appauvrit rapidement en carbone, puis en azote, au cours de la réaction isotherme à $650^{\circ} \mathrm{C}$. En outre, ce mécanisme peut permettre de comprendre la transition $\mathrm{M}_{23} \mathrm{C}_{6}=>\mathrm{M}_{2} \mathrm{X}$ observée au cours de la réaction isotherme à $650^{\circ} \mathrm{C}$. En effet, la figure 6 présente une micrographie électronique de la précipitation apparue après décomposition isotherme de l'austénite à $650^{\circ} \mathrm{C}$ dans un acier $9 \mathrm{Cr} 1 \mathrm{Mo}$ contenant $700 \mathrm{ppm}$ d'azote au lieu de $250 \mathrm{ppm}$ dans l'acier EM10 présenté ici. Cette micrographie souligne une proportion beaucoup plus importante de précipités du type $\mathrm{M}_{2} \mathrm{X}$ dans cet alliage dopé a l'azote, comparativement a l'EM10 pour lequel la phase majoritaire précipitée est $\mathrm{M}_{23} \mathrm{C}_{6}$. Ceci montre que, pour une composition nominale de l'alliage telle que $[\mathrm{C}] /[\mathrm{N}] \approx 1.5$, la phase majoritaire précipité à $650^{\circ} \mathrm{C}$ est $\mathrm{M}_{2} X$, alors que, pour un rapport nominal $[C] /[N] \approx 4$ (EM10), la phase majoritaire est $M_{23} C_{6}$. Il est donc vraisemblable que le rapport $[\mathrm{C}] /[\mathrm{N}]$ en solution solide dans l'austénite non transformée diminue au cours de la réaction isotherme a $650^{\circ} \mathrm{C}$ dans l'EM10. On peut alors penser que la transition $M_{23} C_{6} \Rightarrow>M_{2} X$ intervient a partir du moment où ce rapport tend vers 1 . La figure 7 présente des valeurs de "libres parcours moyens" de plusieurs éléments dans la phase austénitique, calculés a partir de différentes valeurs des coefficients de 
diffusion trouvées dans la littérature pour des matrices à base fer plus ou moins alliées. On peut remarquer, tout d'abord, que l'azote diffuse moins vite dans l'austénite que le carbone (d'un facteur $\approx 5$ ) à $650^{\circ} \mathrm{C}$. Ceci est donc en accord avec le fait que l'austénite puisse s'appauvrir plus vite en carbone qu'en azote a $650^{\circ} \mathrm{C}$. $\mathrm{D}$ 'autre part, il apparait clairement que les atomes substitutionnels $(\mathrm{Fe}, \mathrm{Cr}$ ) diffusent beaucoup plus lentement dans l'austénite que les interstitiels (d'un facteur $\approx 100$ à 1000) à cette température. Ce fait est d'ailleurs à la base du modèle de "paraéquilibre" proposé par HULTGREN et analysé thermodynamiquement par HILLERT ([24] et [25]), ce modele étant généralement utilisé pour décrire la décomposition isotherme de l'austénite en ferrite dans les aciers alliés.

Enfin, la figure 8 présente l'évolution de différents paramètres : la température de début de transformation martensitique (Ms) et la dureté de la martensite correspondant à l'austénite non transformée ainsi que la variation du "pouvoir thermoélectrique" (PTE), en fonction du taux de transformation à 650, 725 et $785^{\circ} \mathrm{C}$. Ces parametres sont tous trois sensibles a la concentration en solution solide des interstitiels (C et $\mathrm{N}$ ) dans l'austénite non transformée à $\theta_{\mathbf{i}}$ (et donc dans la martensite). Les évolutions expérimentales de ces trois parametres semblent bien confirmer l'hypothèse selon laquelle l'austénite s'appauvrit rapidement en atomes interstitiels à $650^{\circ} \mathrm{C}$, ce qui ne semble pas être le cas pour les températures voisines du "nez" du diagramme TTT $\left(725-785^{\circ} \mathrm{C}\right)$.

\section{DECOMPOSITION ANISOTHERME DE L'AUSTENITE LORS DE REFROIDISSEMENTS CONTINUS:}

La figure 9 présente le diagramme TRC de l'EM10 après austénitisation $1 \mathrm{~h} \mathrm{à} 1030^{\circ} \mathrm{C}$ et des micrographies des structures associees pour deux vitesses caractéristiques de refroidissement. On peut remarquer que la "vitesse critique de trempe martensitique" $\left(v_{\text {crit. }}\right)$ est faible $\left(55^{\circ} \mathrm{C} / \mathrm{h}\right)$, soulignant la bonne "trempabilite" de cet acier. Un refroidissement à une vitesse légerement inférieure à vcrit. $\left(50^{\circ} \mathrm{C} / \mathrm{h}\right)$ conduit à l'apparition de quelques germes de ferrite situé prefférentiellement a l'"ex-joint" triple austénitique. A cette vitesse de refroidissement, la transformation \{austénite $\}=>$ ferrite $\}$ au refroidissement s'étend entre $\approx 730^{\circ} \mathrm{C}$ et $675^{\circ} \mathrm{C}$. Afin d'interpréter les microstructures obtenues après refroidissement à $50^{\circ} \mathrm{C} / \mathrm{h}$, on peut décrire le refroidissement dans le domaine de transformation de l'austenite en ferrite par deux paliers isothermes : $1^{\prime}$ un à une temperature superieure a $700^{\circ} \mathrm{C}\left(\approx 715^{\circ} \mathrm{C}\right)$, l'autre à une température inférieure $\left(\approx 685^{\circ} \mathrm{C}\right)$. Dans cette gamme de vitesse de refroidissement et d'après les résultats obtenus en conditions isothermes, on pouvait s'attendre à une germination préférentielle de la ferrite sur les joints triples, car celle-ci a lieu au refroidissement à une température supérieure a $700^{\circ} \mathrm{C}$. D' autre part, le fait que le refroidissement puisse être décrit par deux paliers isothermes, l'un dans le domaine où la précipitation qui accompagne la décomposition isotherme de l'austénite adopte une morphologie perlitique, et l'autre dans le domaine pour lequel la précipitation est de type "interphase", laisse supposer une coexistence des deux types de précipitation lors de ce refroidissement à $50^{\circ} \mathrm{C} / \mathrm{h}$. C'est effectivement ce qui est observé expérimentalement, comme l'illustrent les micrographies correspondant a la vitesse " 1 " de la figure 9 . En outre, les micrographies révelent que la précipitation peut être différente sur les différents interfaces \{ferrite\}-\{martensite\} au sein d'un même grain $\alpha$. D'après HONEYCOMBE \& al.[19], on peut supposer qu'une interface $\{\alpha\}-\{m\}$ incohérente, de forte énergie, favorise une précipitation de type "perlitique", alors qu'une interface semi-cohérente, de faible énergie, favorise un mode de précipitation de type "interphase". Enfin, si l'on s'intéresse à un refroidissement lent $\left(\mathrm{v}_{2} \approx 10^{\circ} \mathrm{C} / \mathrm{h}\right)$ dans le domaine où l'on obtient une décomposition complete de l'austénite en ferrite, on observe uniquement une structure ferritique avec précipitation à morphologie "perlitique". Ceci était prévisible compte tenu du fait que, pour ces vitesses lentes, la transformation \{austénite $\}=>$ \{ferrite\} au refroidissement $s^{\prime}$ étend sur un domaine étroit de températures situé au dessus de $700^{\circ} \mathrm{C}$. 


\section{CONCLUSIONS:}

Sans vouloir résumer les résultats et les déductions précédentes, il convient d'insister sur l'intérêt que présente l'étude des transformations de phases en conditions isothermes pour pouvoir décrire et mieux appréhender les cinétiques de transformation de phases anisothermes (au refroidissement) et les évolutions structurales associées, dans ces aciers relativement complexes.

L'étude microstructurale a notamment montré que, pour l'acier étudié, la transformation isotherme \{austénite $\}=>$ ferrite\} à températures inférieures à $700^{\circ} \mathrm{C}$ aurait lieu avec un appauvrissement rapide de l'austénite en carbone des le début de la réaction idotherme. Ceci se traduit par une transition de la nature de la précipitation qui accompagne la réaction isotherme : la précipitation des carbures "interphases" $\mathrm{M}_{23} \mathrm{C}_{6}$ fait place à la précipitation de carbonitrures $\mathrm{M}_{2} \mathrm{X}$ au delà de $\approx 20 \%$ d'austénite transformée à $\approx 650^{\circ} \mathrm{C}$. Cette transition pourrait être due a la modification du rapport des concentrations en carbone et azote en solution solide dans l'austénite au cours de la réaction isotherme.

\section{REFERENCES BIBLIOGRAPHIQUES :}

[1] S. J. SANDERSON, Ferritic steels for fast reactor steam generators, BNES, London, 1978, pp. 120-127

[2] M. K. BOOKER \& al., Proc. ASM Int. Conf. on Ferritic Steels for High Temperature Applications, Warrendale, PA, Oct. 6-8, 1981, Ashok K., ed., ASM, Metals Park, OH, pp. 257-273

[3] F B PICKERING \& al., Metals Technology, Oct. 1980, pp. 409-413

[4] B A SENIOR \& al., Acta Met., 1988, Vol. 36, No7, pp. 1855-1862

[5] A. ALAMO \& al., Mem. Et. Sci. Rev. Met., Octobre 1990

[6] J-C BRACHET \& al., Mem. Et. Sci. Rev. Met., Janv. 90, pp. 33-46

[7] J-C. BRACHET, Rapport CEA R-5581, France, 1991

[8] J-C. BRACHET, Proceeding of the 8th. Int. Congress on the Heat Treatment of Mat., I. TAMURA Ed., KYOTO, Nov. 1992, pp. 89-93

[9] R. L. KLUEH \& al., JNM 182, 1991, pp. 230-239

[10] W B JONES \& al., Met. Trans., May 1991, Vol. 22A, pp. 1049-1058

[11] K. TOKUNO \& al., Scripta Met. \& Mat., 1991, Vol. 25, pp. 1763-1768

[12] K.R. KINSMAN \& H.I. AARONSON, Transformation and Hardenability in Steels, Climax Molybdenum Co, 1967, p. 39

[13] P. W. BACH \& al., Scripta Met., Vol. 14, 1980, pp. 205-210

[14] I. STARK \& al., Phase transformation '87, G. LORIMER, ed., Inst. of Metals, London, 1988, pp. 475-481

[15] H. I. AARONSON \& al., Met. trans., Vol. 21A, June 1990, pp. 1413-1491

[16] W A JONHSON \& R F MEHL, Trans. AIME, 1939, 135, p. 416

[17] J. BURKE, la Cinétique des Changements de Phase dans les Métaux, Ed. Masson \& Cie, 1968

[18] F. G. BERRY \& R. W. K. HONEYCOMBE, Met. Trans. Vol. 1, Déc. 1970, pp. 3279-3286

[19] R. W. K. HONEYCOMBE, Met. Trans. Vol. 7A, Juillet 1976, pp. 915-936

[20] J. V. BEE \& R. W. K. HONEYCOMBE, Met. Trans., Vol. 9A, Avril 1978, pp. 587-593

[21] P. R. HOWELL \& al., Met. Trans., vol. 10A, Sept. 1979, pp. 1213-1222

[22] J. W. CHRISTIAN, The Theory of Transformation in Metals and Alloys, Pergamon Press, 2 nd. Ed., 1975

[23] J. W. CAHN, Acta met., Vol. 4, Sept. 1956, pp. 449-459

[24] A. HULTGREN, Trans. ASM, Vol. 39, 1947, p. 915

[25] M. HILLERT, The Mechanism of Phase Transformations in Crystalline Solid, session IV, Inst. of Met., London, 1969 , p. 23 


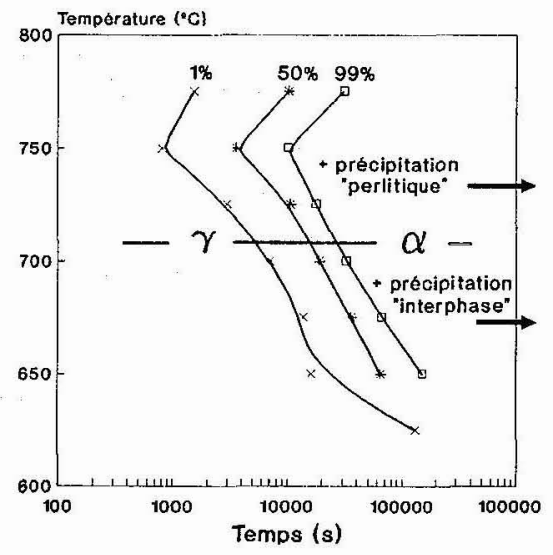

Fig. 1 : Diagramme TTT de l'acier 9Cr1Mo (EM10) après austénitisation $1 \mathrm{~h} 1030^{\circ} \mathrm{C}$.
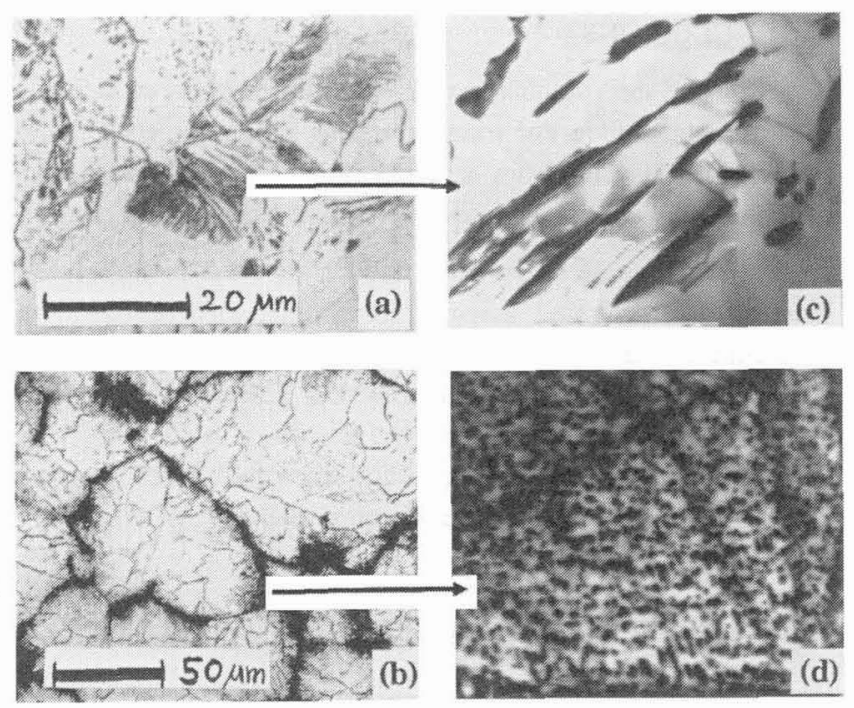

Fig. 1-bis : Micrographies optiques $\{(\mathbf{a}),(\mathrm{b})\}$ et électroniques (MET) sur lame mince $\{(\mathrm{c}),(\mathrm{d})\}$.

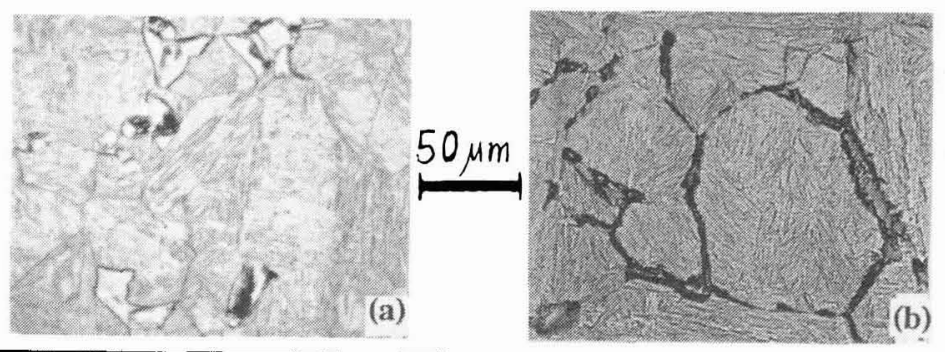

Fig. 2 : Micrographies optiques des structures obtenues après transformation isotherme partielle de l'austénite en ferrite $\left(Y(\alpha) \approx 10 \%\right.$ ), a $725^{\circ} \mathrm{C}$ (a) et $650^{\circ} \mathrm{C}$ (b).

Nb. grains $\alpha$ / Surf.

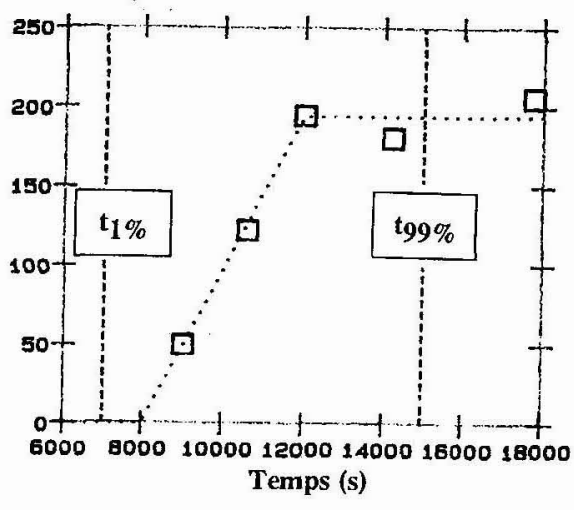

Fig. 3 : Variation du nombre de grains de ferrite apparus / surface analysée $\left(0.18 \mathrm{~mm}^{2}\right)$, en fonction du temps de réaction isotherme a $725^{\circ} \mathrm{C}$.

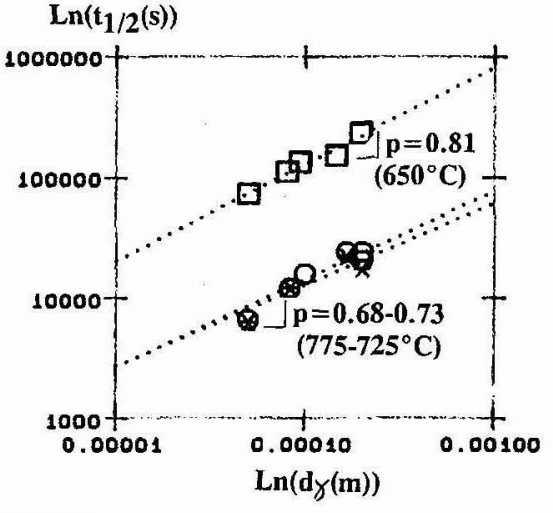

Fig. 4 : Variation du temps de demi-réaction à $650^{\circ} \mathrm{C}, 725^{\circ} \mathrm{C}$ et $775^{\circ} \mathrm{C}$, en fonction du diamètre moyen du grain austénitique. 

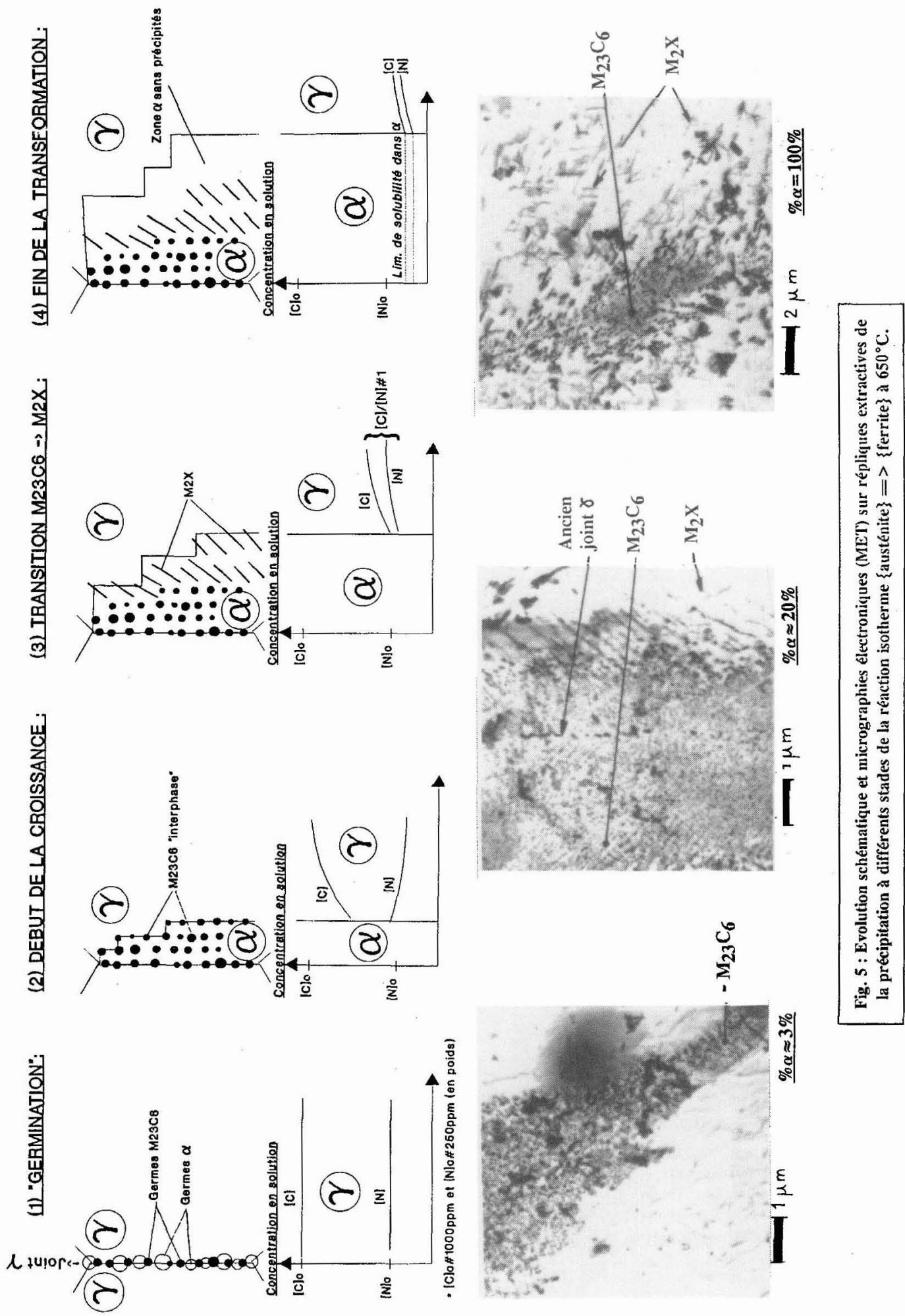


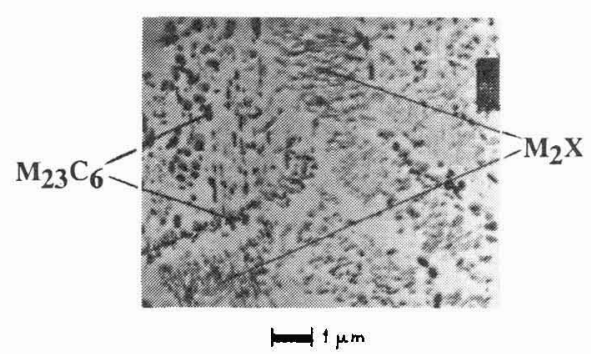

Fig. 6 : Micrographie électronique (MET) sur réplique de la précipitation apparue après decomposition isotherme totale de l'austénite à $650^{\circ} \mathrm{C}$ (alliage $9 \mathrm{Cr} 1 \mathrm{MoN}$ ).

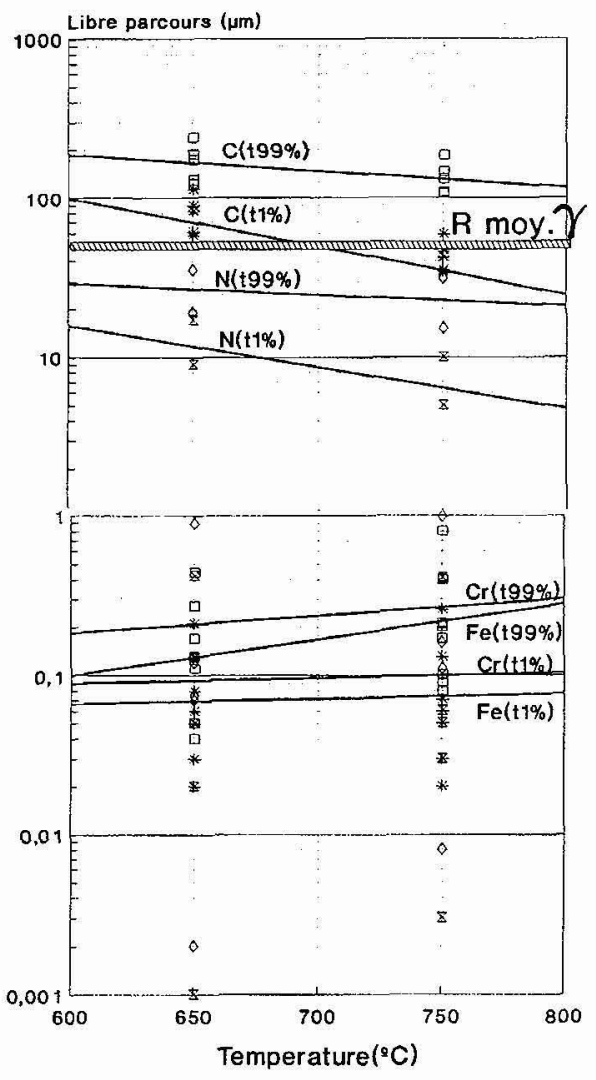

Fig. 7 : Libres parcours moyens des atomes $\mathrm{C}, \mathrm{N}, \mathrm{Fe}$ et $\mathrm{Cr}$ au bout des temps caractéristiques (tI\% et $t 99 \%$ ) de transformation isotherme a $650^{\circ} \mathrm{C}$ et à $750^{\circ} \mathrm{C}$.

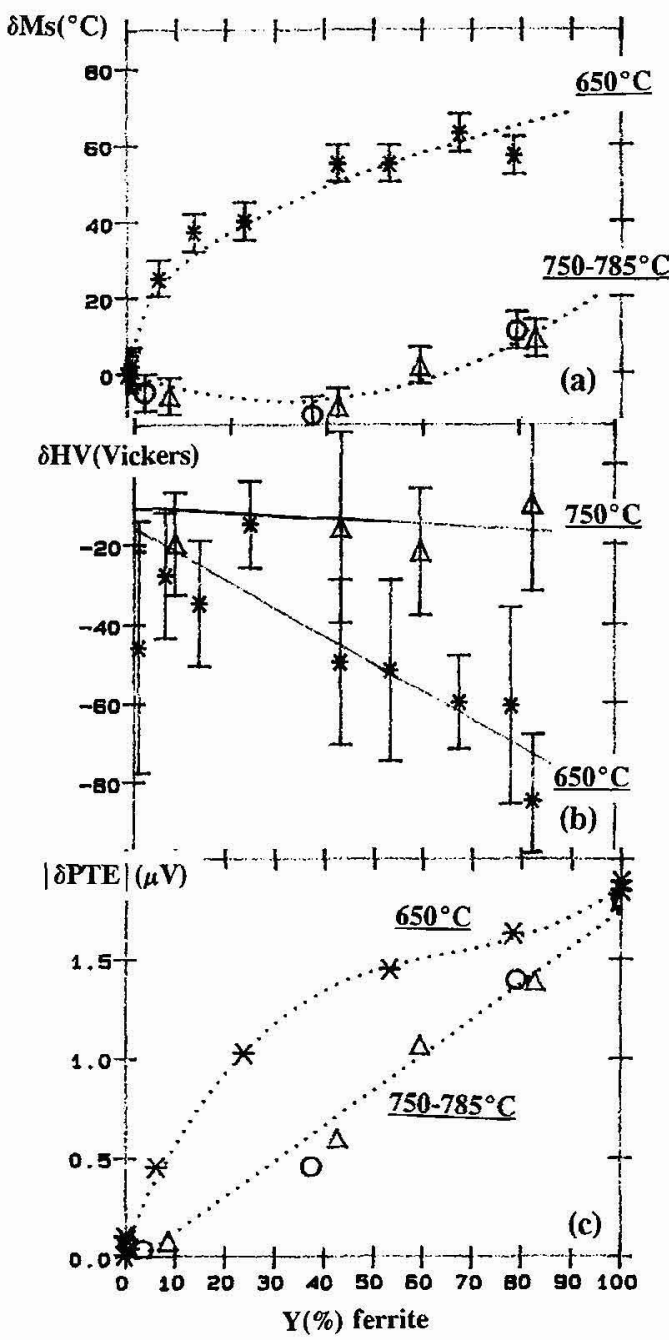

Fig. 8 : Variation de la température de début de la transformation martensitique (a), de la dureté Vickers $(0.3 \mathrm{Kg}$ ) de la martensite (b) et du pouvoir thermoelectrique (PTE) (c), en fonction du taux de transformation isotherme a $650^{\circ} \mathrm{C}, 750^{\circ} \mathrm{C}$ et $785^{\circ} \mathrm{C}$. 
C3-91

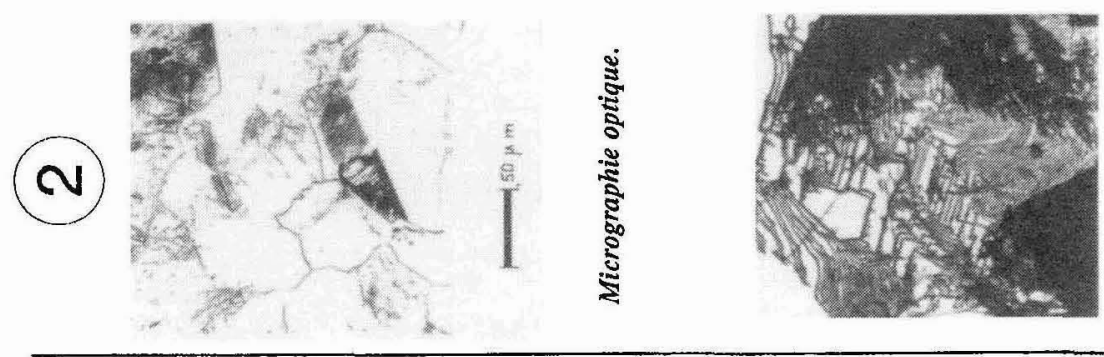

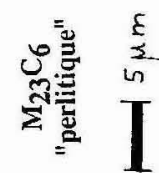
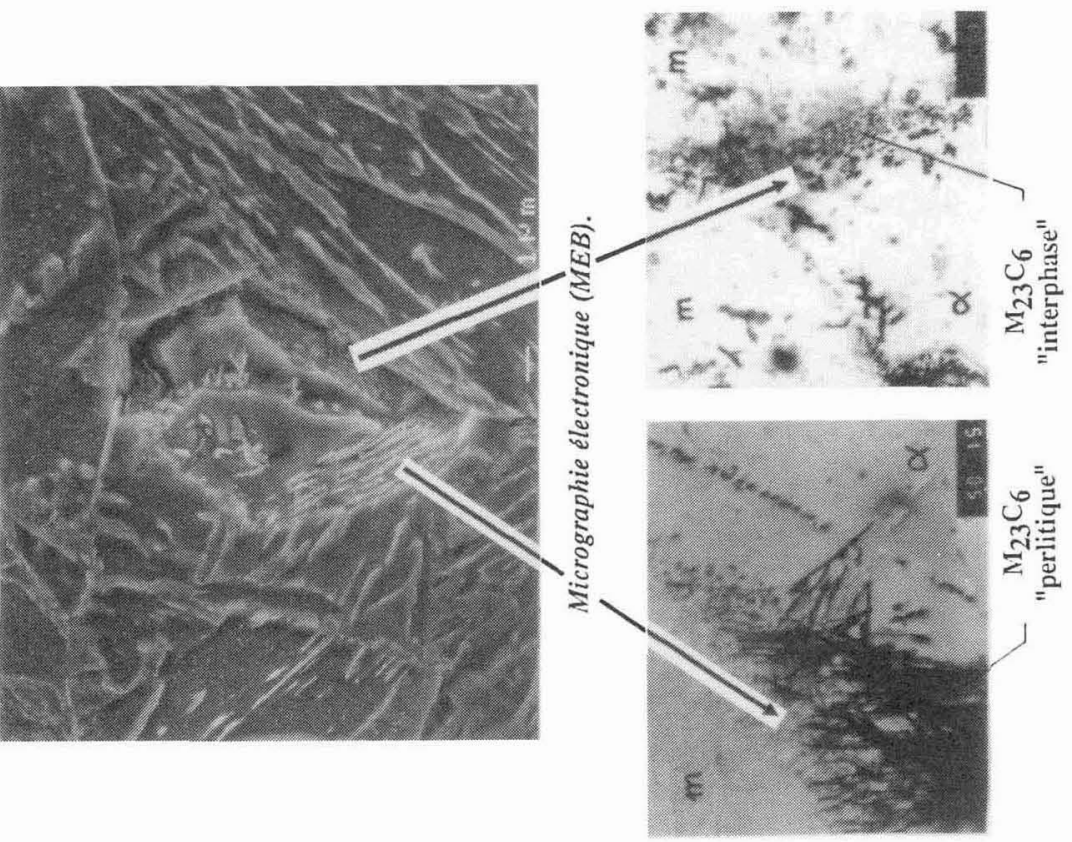

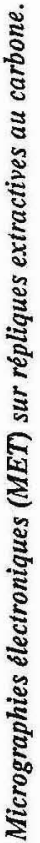

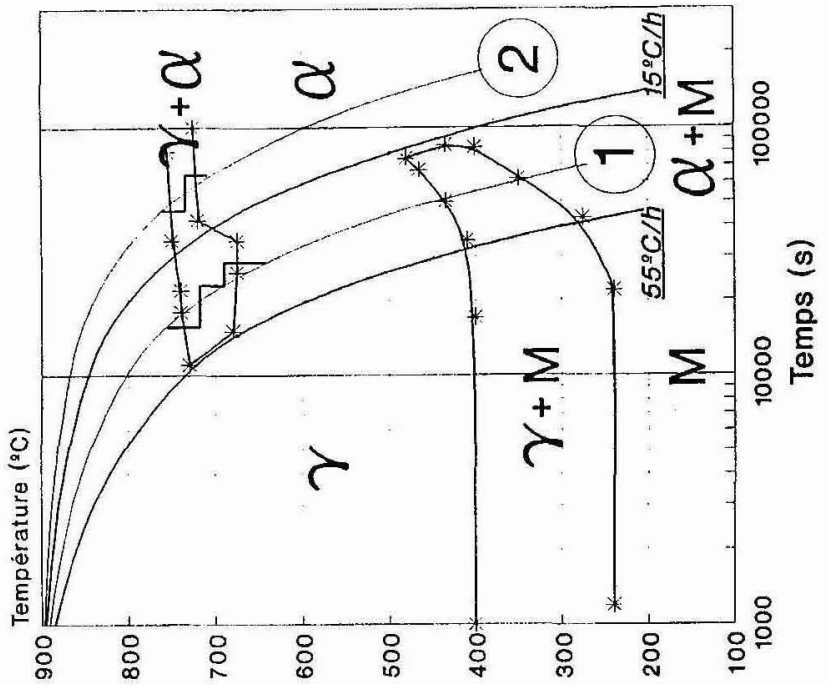

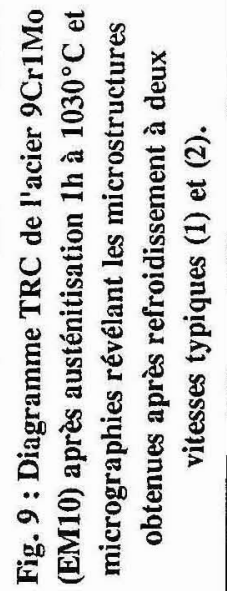

